\title{
Acute effects of apple and blackcurrant polyphenol-rich extracts on postprandial glycaemia and vascular function in healthy men and women
}

\author{
M.L. Castro-Acosta, S.G. Stone, J.E. Mok, R.K. Mhajan, C-I. Fu, G.N. Lenihan-Geels and \\ W.L. Hall \\ Diabetes \& Nutritional Sciences Division, Faculty of Life Sciences and Medicine, King's College London, \\ 150 Stamford Street, London, SE1 9NH, UK
}

Elevated postprandial glucose concentrations lead to endothelial dysfunction. Certain fruit polyphenols (e.g. phloridzin, anthocyanins) inhibit intestinal glucose transport ${ }^{(1)}$. Others (e.g. anthocyanins, proanthocyanidins, ellagitannins) reduce starch- and sucrosedigesting enzyme activity ${ }^{(2)}$. The effects of consumption of apple polyphenols on postprandial glucose concentrations following starch/sucrose-containing meals have not been fully characterised. The aim of this study was to determine whether postprandial glycaemia and vascular function following high-carbohydrate meals were influenced by a polyphenol-rich apple extract, with and without an anthocyanin-rich blackcurrant extract.

Twenty-five healthy subjects (20 M, 5 postmenopausal W, mean age $32 \mathrm{y}$, SD 14) completed a randomised, double-blind, crossover study. Three matched fruit cordial drinks contained either 1) $1.2 \mathrm{~g}$ apple polyphenols (A); 2) $0.6 \mathrm{~g}$ apple polyphenols $+0.6 \mathrm{~g}$ blackcurrant anthocyanins (1.4 g blackcurrant polyphenols) (AB); or, 3) no added polyphenols (CON; control). The drink (12.2 g carbohydrate, $195 \mathrm{~kJ})$ was administered immediately before a high-carbohydrate meal (41 g starch, $22 \mathrm{~g}$ sucrose, $1.3 \mathrm{MJ})$. Venous plasma glucose was measured at baseline and frequent time-points up to $2 \mathrm{~h}$, and digital volume pulse stiffness index (DVP-SI) and reflection index (DVP-RI) were assessed at baseline and $1,1 \frac{1}{2}$ and $2 \mathrm{~h}$.

Plasma glucose total iAUC 0-120 min $(P<0.05)$ and early iAUC $0-30 \mathrm{~min}$, Cmax and Tmax were reduced by treatments A and AB compared with CON (all $P<0 \cdot 0001$ ). Mean differences in iAUC 0-30 min were: A - CON, $-25 \cdot 3 \mathrm{mmol} / \mathrm{L} \cdot \mathrm{min}(95 \% \mathrm{CI}-33 \cdot 6$, -16.9); AB - CON, $-33.1 \mathrm{mmol} / \mathrm{L} \cdot \min (95 \% \mathrm{CI}-42 \cdot 4,-23 \cdot 8)$; AB - A, $-7.8 \mathrm{mmol} / \mathrm{L} \cdot \mathrm{min}(95 \% \mathrm{CI}-12 \cdot 5,-3 \cdot 2)$. The overall treatment effect was significant for changes in DVP-RI $(P=0.015)$, a parameter of vascular function which is indicative of small- to medium-sized arterial stiffness and influenced by peripheral arterial vasodilation. DVP-RI significantly decreased following A compared to $\mathrm{CON}$ but the decrease following $\mathrm{AB}$ was not statistically significantly different from CON (mean differences in $\triangle \mathrm{DVP}-\mathrm{RI}$ (\% units)): A-CON, -6\% (95\% CI - 11, - 2); AB-CON, -3\% (95\% CI -8, 1); AB-A, $3 \%(95 \%$ CI -4, 10). There were no significant effects of treatment on DVP-SI, an indicator of stiffness of large elastic arteries.

In conclusion, consumption of $1.2 \mathrm{~g}$ apple polyphenols reduced plasma glucose concentrations following a starch and sucrosecontaining meal and prevented the postprandial increase in pulse wave reflection associated with postprandial glycaemia. Although the lower dose of apple polyphenols (plus blackcurrant polyphenols) had a slightly larger inhibitory effect on glycaemic response, there was no significant amelioration of postprandial vascular dysfunction. These findings suggest that apple polyphenol metabolites absorbed within $2 \mathrm{~h}$ of ingestion may have been primarily responsible for the observed improvements in postprandial vascular function within this timeframe.

1. Schulze C, Bangert A, Kottra G, et al. (2014) Mol Nutr Food Res 58, 1795-808.

2. Williamson G. (2012) Mol Nutr Food Res. 57, 48-57. 